\title{
MONOTONIC SEMICONJUGACIES ONTO EXPANDING MAPS OF THE INTERVAL ${ }^{1}$
}

\author{
BILL BYERS
}

\begin{abstract}
A contraction mapping is used to produce a semiconjugacy from a map $\tau_{1}$ with maximum at $c_{1}$ to an expanding unimodal map with maximum at $c_{2}$ under the assumption that there is an interval $J$ containing $c_{1}$ such that there is a one-to-one, order-preserving correspondence between the orbit of $J$ under $\tau_{1}$ and the orbit of $c_{2}$ under the expanding map.
\end{abstract}

Introduction. In [5] Milnor and Thurston prove the existence of semiconjugacies from a given piecewise monotonic map, $\tau_{1}$, with growth number $s>1$, onto an (expanding) map of constant slope $s$. This semiconjugacy is exploited in [4] to get a "spectral decomposition" of the nonwandering set of such maps. In [1] it is shown that any piecewise monotonic map with unique itineraries can be used as a "model" for other piecewise monotonic maps with the same kneading sequence if the orbits of the various turning points are infinite and disjoint. All of the above rely heavily on the machinery of "kneading sequences" developed by Milnor and Thurston. In this note we consider expanding unimodal maps $\tau_{2}$. Using simple function space methods we show that such maps are "models" for various classes of maps $\tau_{1}$ which possess a closed interval whose orbit is in a one-to-one, order-preserving correspondence with the orbit of the turning point of $\tau_{2}$.

Discussion. Consider a pair of maps $\tau_{1}, \tau_{2}:[0,1] \rightarrow[0,1]$ satisfying the following conditions:

(1) $\tau_{1}$ is piecewise monotonic but not necessarily continuous; i.e. there exists a point $0<c_{1}<1$ such that $\tau_{1}$ is nondecreasing on $\left[0, c_{1}\right]$ and nonincreasing on $\left[c_{1}, 1\right]$.

(2) $\tau_{2}$ is unimodal, continuous, and expanding; i.e. $\tau_{2}$ is strictly increasing on $\left[0, c_{2}\right]$ and strictly decreasing on $\left[c_{2}, 1\right]$ and in addition there exists a constant $\lambda>1$ such that $\left|\tau_{2}(x)-\tau_{2}(y)\right| \geqslant \lambda|x-y|$, for $x$ and $y$ in either subinterval. Also, we shall always have $\tau_{2}(0)=\tau_{2}(1)=0$.

THEOREM 1. Suppose $\tau_{1}, \tau_{2}$ satisfy (1) and (2) above and in addition there exists a closed interval $J$ ( possibly degenerate) containing $c_{1}$ such that either

(3) the intervals $\tau_{1}^{n}(J)$ are disjoint for $n=0,1,2, \ldots$ and the images $\tau_{2}^{n}\left(c_{2}\right)$ are all distinct for $n=0,1, \ldots$ or

Received by the editors August 24, 1981 and, in revised form, December 8, 1982.

1980 Mathematics Subject Classification. Primary 54H20; Secondary 58F99.

${ }^{1}$ Research supported by NSERC grant \# A-5325 and an FCAC grant from the Quebec Department of Education. 
(3') the intervals $\tau_{1}^{i}(J)$ are disjoint for $i=0,1, \ldots, n+k-1$ but $\tau_{1}^{n+k}(J) \subset \tau_{1}^{k}(J)$. Similarly the points $\tau_{2}^{i}\left(c_{2}\right)$ are all distinct for $i<n+k$ but $\tau_{2}^{n+k}\left(c_{2}\right)=\tau_{2}^{k}\left(c_{2}\right)$. In either case we also assume

(4) $\tau_{1}^{i}(J)<\tau_{1}^{j}(J) \Leftrightarrow \tau_{2}^{i}\left(c_{2}\right)<\tau_{2}^{j}\left(c_{2}\right)$.

There then exists a unique monotonically increasing map $\psi:[0,1] \rightarrow[0,1]$ such that $\psi \circ \tau_{1}=\tau_{2} \circ \psi$ and $\psi\left(\left[0, c_{1}\right]\right) \subseteq\left[0, c_{2}\right] ; \psi\left(\left[c_{1}, 1\right]\right) \subseteq\left[c_{2}, 1\right]$.

Proof. Let $\Psi$ be the set of nondecreasing functions $\psi:[0,1] \rightarrow[0,1]$ such that $\psi\left(\tau_{1}^{i}(J)\right)=\tau_{2}^{i}\left(c_{2}\right)$ for all $i$. Now $\Psi$ is a complete metric space when it is given the uniform metric. It is clearly nonempty because of (4). For $\psi \in \Psi$ define $T(\psi)$ by

$$
T \psi(x)= \begin{cases}\tau_{2,1}^{-1}\left(\psi\left(\tau_{1}(x)\right)\right) & \text { for } x \in\left[0, c_{1}\right], \\ \tau_{2,2}^{-1}\left(\psi\left(\tau_{1}(x)\right)\right) & \text { for } x \in\left[c_{1}, 1\right],\end{cases}
$$

where $\tau_{2,1}=\tau_{2} \mid\left[0, c_{2}\right]$ and $\tau_{2,2}=\tau_{2} \mid\left[c_{2}, 1\right]$.

Now for any $x, \psi\left(\tau_{1}(x)\right) \leqslant \psi\left(\tau_{1}\left(c_{1}\right)\right)=\tau_{2}\left(c_{2}\right)$ since $\psi$ is monotonic. Thus $\tau_{2, i}^{-1}$ $(i=1,2)$ and therefore $T(\psi)(x)$ are defined. Moreover, $\tau_{2, i}^{-1}\left(\psi\left(\tau_{1}\left(c_{1}\right)\right)\right)=\tau_{2, i}^{-1}\left(\tau_{2}\left(c_{2}\right)\right)$ $=c_{2}$ for $i=1,2$. Thus $T \psi\left(c_{1}\right)=c_{2}$. To check that $T(\psi) \in \Psi$ notice that $T(\psi)$ is monotone by construction since $\tau_{1}$ and $\tau_{2}$ are piecewise monotone. To verify that $T(\psi)\left(\tau_{1}^{i} J\right)=\tau_{2}^{i}\left(c_{2}\right)$, suppose, for example, that $\tau_{1}^{i}(J)<c_{1}$. Then $T(\psi)\left(\tau_{1}^{i}(J)\right)=$ $\tau_{2,1}^{-1}\left(\psi\left(\tau_{1}^{i+1}(J)\right)\right)=\tau_{2,1}^{-1}\left(\tau_{2}^{i+1}\left(c_{2}\right)\right)$. But $\tau_{1}^{i}(J)<c_{1} \Leftrightarrow \tau_{2}^{i}\left(c_{2}\right)<c_{2}$ by (4). Thus $\tau_{2,1}^{-1}\left(\tau_{2}\left(\tau_{2}^{i}\left(c_{2}\right)\right)\right)=\tau_{2}^{i}\left(c_{2}\right)$

Moreover $T$ is a contraction mapping since

$$
\left|T \psi_{1}-T \psi_{2}\right|=\sup _{x}\left|T \psi_{1}(x)-T \psi_{2}(x)\right|
$$

and

$$
\begin{aligned}
\sup _{x \leqslant c_{1}}\left|T \psi_{1}(x)-T \psi_{2}(x)\right| & =\sup _{0 \leqslant x \leqslant c_{1}}\left|\tau_{2,1}^{-1} \psi_{1} \tau_{1}(x)-\tau_{2,1}^{-1} \psi_{2} \tau_{1}(x)\right| \\
& \leqslant\left(\sup _{0 \leqslant x \leqslant c_{1}}\left|\psi_{1} \tau_{1}(x)-\psi_{2} \tau_{1}(x)\right|\right) / \lambda \\
& \leqslant\left(\sup _{y}\left|\psi_{1}(y)-\psi_{2}(y)\right|\right) / \lambda \\
& =\left(\left|\psi_{1}-\psi_{2}\right|\right) / \lambda .
\end{aligned}
$$

Similarly, $\sup _{c_{1} \leqslant x \leqslant 1}\left|T \psi_{1}(x)-T \psi_{2}(x)\right| \leqslant\left(\left|\psi_{1}-\psi_{2}\right|\right) / \lambda$.

Thus $T$ has a unique fixed point $\psi_{0}$. It is clear that $\psi_{0}$ satisfies $\tau_{2} \circ \psi_{0}=\psi_{0} \circ \tau_{1}$ and $\psi_{0}\left(\left[0, c_{1}\right]\right) \subseteq\left[0, c_{2}\right], \psi_{0}\left(\left[c_{1}, 1\right]\right) \subseteq\left[c_{2}, 1\right]$. It is straightforward to show that any map $\psi$ satisfying the above conditions is a fixed point of $T$.

COROLlARY 1. If $\tau_{1}$ is continuous then the semiconjugacy $\psi_{0}$ is also continuous.

Proof. The set $C$ of continuous monotone functions in $\Psi$ is closed and invariant under $T$. Thus it suffices to show that $\mathcal{C}$ is nonempty. That is we must show that the correspondence $\tau_{1}^{i}(J) \mapsto \tau_{2}^{i}\left(c_{2}\right)$ extends to a continuous map on the closure of the 
union of the $\tau_{1}^{i}(J)$. For then we can extend linearly on each complementary interval. Under condition (3') this is immediate. Under condition (3), it clearly suffices to show that for any point $x$ such that

$$
\tau_{1}^{n_{k}}(J) \uparrow x \quad \text { and } \quad \tau_{1}^{m_{k}}(J) \downarrow x,
$$

the corresponding limits for the sequences $\left\{\tau_{2}^{n_{k}}\left(c_{2}\right)\right\}$ and $\left\{\tau_{2}^{m_{k}}\left(c_{2}\right)\right\}$ are equal. That is, if we set $A=\lim _{k \rightarrow \infty} \tau_{2}^{n_{k}}\left(c_{2}\right)$ and $B=\lim _{k \rightarrow \infty} \tau_{2}^{m_{k}}\left(c_{2}\right)$, then $A=B$.

Suppose $A<B$ and $I=[A, B]$. If $c_{2} \notin \tau_{2}^{n}(I)$ for $n=0,1,2, \ldots$, then the length of $\tau_{2}^{n}(I)$ is greater or equal to $\lambda^{n}$ times length of $I$ (condition (2)). This is clearly impossible and so we may suppose that $c_{2} \notin \tau_{2}^{k}(I), k=0,1, \ldots, n-1$, and $c_{2} \in$ $\tau_{2}^{n}(I)$. Therefore $\tau_{2}^{n} \mid I$ is a homeomorphism. Suppose for definiteness that it is increasing. Then $\tau_{2}^{n+n_{k}}\left(c_{2}\right)$ increases to $\tau_{2}^{n}(A)$ and $\tau_{2}^{n+m_{k}}\left(c_{2}\right)$ decreases to $\tau_{2}^{n}(B)$ (for large $k$ ) where $\tau_{2}^{n}(A) \leqslant c_{2} \leqslant \tau_{2}^{n}(B)$. Therefore by (4) we have

$$
\tau_{1}^{n+n_{h}}(J) \leqslant J \leqslant \tau_{1}^{n+m_{h}}(J) \quad(\text { for large } k),
$$

but $\lim _{k \rightarrow \infty} \tau_{1}^{n+n_{k}}(J)=\tau_{1}^{n}(x)=\lim _{k \rightarrow \infty} \tau_{1}^{n+m_{k}}(J)$ and so $J=\tau_{1}^{n}(x)$. Thus $J=$ $\left\{c_{1}\right\}=\left\{\tau_{1}^{n}(x)\right\}$.

Therefore we can reduce the question to a consideration of the case where $\tau_{1}^{p_{k}}\left(c_{1}\right) \uparrow c_{1}, \tau_{1}^{q_{k}}\left(c_{1}\right) \downarrow c_{1}$ and $\lim _{k \rightarrow \infty} \tau_{2}^{p_{k}}\left(c_{2}\right)=A, \lim _{k \rightarrow \infty} \tau_{2}^{q_{k}}\left(c_{2}\right)=B$ where $A \leqslant c_{2}$ $\leqslant B$ and we wish to show $A=B$. Assume $A<c_{2}$ and let $I=\left[A, c_{2}\right]$. As above we may assume that $c_{2}$ does not belong to the interior of $\tau_{2}^{k}(I), k=0, \ldots, n-1$, and $c_{2}$ belongs to the interior of $\tau_{2}^{n}(I)$. Reasoning exactly as above, we conclude that $\tau_{1}^{n}\left(c_{1}\right)=c_{1}$. This contradicts $\left(3^{\prime}\right)$ and completes the proof.

Note. If $\tau_{1}(0)=\tau_{1}(1)=0$, then the fixed point $\psi_{0}$ must have $\psi_{0}(0)=0$ and $\psi_{0}(1)=1$. Thus in the above corollary $\psi_{0}$ is onto.

THEOREM 2. Suppose $\tau_{1}, \tau_{2}$ satisfy conditions (2), (3') and (4) of Theorem 1 but in place of piecewise monotonicity of $\tau_{1}$ we assume

(1') $\tau_{1}$ is continuous with $\tau_{1}(x) \leqslant \tau_{1}\left(c_{1}\right)$ for all $x$ and if $J$ is the closed interval of condition (4) we assume that if $\tau_{1}^{i}(J)<\tau_{1}^{j}(J)$ are adjacent (i.e. there is no $k$ with $\left.\tau_{1}^{i}(J)<\tau_{1}^{k}(J)<\tau_{1}^{j}(J)\right)$, then $\tau_{1}$ maps the closed interval between $\tau_{1}^{i}(J)$ and $\tau_{1}^{j}(J)$ onto the interval between $\tau_{1}^{i+1}(J)$ and $\tau_{1}^{j+1}(J)$.

Then the conclusion of Corollary 1 holds.

Proof. The proof proceeds as in Theorem 1 except that we modify $\Psi$ as follows: $\psi \in \Psi \Leftrightarrow \psi$ is continuous, $\psi\left(\tau_{1}^{i}(J)\right)=\tau_{2}^{i}\left(c_{2}\right)$, and $\psi$ maps the closed interval bounded by the adjacent intervals $\tau_{1}^{i}(J)<\tau_{1}^{j}(J)$ onto $\left[\tau_{2}^{i}\left(c_{2}\right), \tau_{2}^{j}\left(c_{2}\right)\right]$. We need only check that $T(\Psi) \subseteq \Psi$. If $\psi \in \Psi, x \in[0,1]$ then $\tau_{1}(x) \leqslant \tau_{1}\left(c_{1}\right)$ and so $\psi \tau_{1}(x) \leqslant \tau_{2}\left(c_{2}\right)$ by $(1)^{\prime \prime}$. Thus $T \psi(x)$ is well defined. Finally $T(\psi)$ is continuous and if $\tau_{1}^{i}(J)<\tau_{1}^{j}(J)<$ $c_{1}$ (for example) then $\tau_{1}^{i+1}(J)<\tau_{1}^{j+1}(J)$ thus

$$
(T(\psi))\left(\left[\tau_{1}^{i}(J), \tau_{2}^{j}(J)\right]\right)=\tau_{2,1}^{-1}\left(\left[\tau_{2}^{i-1}\left(c_{2}\right), \tau_{2}^{j-1}\left(c_{2}\right)\right]\right)=\left[\tau_{2}^{i}\left(c_{2}\right), \tau_{2}^{j}\left(c_{2}\right)\right]
$$

using conditions (4) and $\left(1^{\prime}\right)$. Thus $T(\psi) \in \Psi$ and the theorem follows. 
EXAMPLE. Suppose $\tau_{1}:[0,1] \rightarrow[0,1]$ is any continuous, onto map with $\tau_{1}(0)=\tau_{1}(1)$ $=0$. If $\tau_{1}(c)=1$, there exists a unique continuous semi-conjugacy $\psi$ from $\tau_{1}$ to the triangle map

$$
\tau_{2}(x)= \begin{cases}2 x, & 0 \leqslant x \leqslant \frac{1}{2}, \\ 2(1-x), & \frac{1}{2} \leqslant x \leqslant 1,\end{cases}
$$

such that $\psi([0, c]) \subseteq\left[0, \frac{1}{2}\right]$ and $\psi\left(\left[c_{1}, 1\right]\right) \subseteq\left[\frac{1}{2}, 1\right]$.

Note. The solution to the conjugacy equation need not be unique in general. Consider the Chebyshev polynomials $T_{n}(x)=\cos \left(n \cos ^{-1} x\right)$ from the closed interval $[-1,1]$ into itself. For example $T_{2}(x)=2 x^{2}-1$ and $T_{3}(x)=4 x^{3}-3 x$. It is well know that $T_{2}$ is topologically conjugate to the triangle map $\tau(x)=2|x|-1$. Suppose $\psi$ is the conjugacy i.e. $\psi \circ T_{2}=\tau \circ \psi$. Since $T_{2} \circ T_{n}=T_{n} \circ T_{2}$ for $n=$ $2,3, \ldots$, we have $\left(\psi \circ T_{n}\right) \circ T_{2}=\psi \circ T_{2} \circ T_{n}=\tau \circ\left(\psi \circ T_{n}\right)$ and $\psi \circ T_{n}$ also satisfies the conjugacy equation for $n=2,3, \ldots$ Our uniqueness results are unaffected, however, since in Theorem 1 we required the semiconjugacy to be monotonic whereas in Theorem 2 we required the semiconjugacy to preserve the intervals about the turning point (here $x=0$ ). However $\psi \circ T_{n}$ maps $[-1,0]$ onto all of $[-1,1]$.

Acknowledgement. I would like to thank Professor A. Boyarsky for some helpful conversations and the referee for his comments.

\section{REFERENCES}

1. B. Byers, Topological semiconjugacy of piecewise monotonic maps of the interval, Trans. Amer. Math. Soc. 276 (1983), 489-495.

2. P. Collet and J. P. Eckmann, Iterated maps on the interval as dynamical systems, Birkhäuser, Boston, Mass., 1980.

3. J. Guckenheimer, Sensitive dependence to initial conditions for one dimensional maps, Comm. Math. Phys. 70 (1979), 133-160.

4. L. Jonker and D. Rand, Bifunctions in one dimension. I: The non-wandering set, Invent. Math. 62 (1981), 347-365.

5. J. Milnor and W. Thurston, On iterated maps of the interval. I, II, Princeton Univ. Press, Princeton, N. j., 1977, preprint.

Department of Mathematics, Concordia University, 7141 Sherbrooke St. West, Montreal, Quebec, CANADA H4B 1R6 